\title{
Análise termodinâmica e \\ cinética de reações de mecanismo de ação para Compostos de Platina (II) Análogos a cisplatina com ligantes derivados de terpenos.
}

\author{
Lucas C. Santana \& Juliana F. Lopes
}

\section{Introdução}

A cisplatina é uma das drogas mais eficientes e utilizadas para o tratamento do câncer1. Entretanto oferece alguns problemas, sendo baixa solubilidade e efeitos colaterais indesejados como: nefrotoxicidade, cardiotoxicidade, ototoxicidade, toxidez ao trato intestinal, entre outros2. Atualmente há mais de 3000 complexos análogos à cisplatina, porém nenhum está à altura da cisplatina3. Por esse motivo há uma busca por complexos de platina (II), a fim de que suas propriedades sejam melhoradas em relação à cisplatina e nesse contexto o estudo tem como principal objetivo utilizar a química computacional para determinar propriedades como: geometria, estabilidade energética, lipofilicidade e barreira de ativação na reação de substituição dos ligantes cloro por ligantes aquo de 12 complexos análogos a cisplatina.

\section{Metodologia}

Todos os cálculos foram realizados nos computadores do LaQC, através do software Gaussian 09, com a metodologia DFT, funcional M062X, função de base para os átomos leves $6,31 \mathrm{~g}(\mathrm{~d}, \mathrm{p})$ e pseudo potencial LanL2DZ para o átomo de platina. Os 12 complexos estudados foram divididos em três modelos para a facilitação em sua identificação. De acordo com a Fig. 1 .<smiles>[R]N1CCN[P+]1(Cl)Cl</smiles><smiles>[R]N1CCCN[P+]1(Cl)Cl</smiles><smiles>[R]N1CCN[P+]1(Cl)Cl</smiles>

Figura 1 - Representação simplificada dos 12 complexos estudados em três modelos base $\left(\mathrm{R}=\mathrm{C}_{5} \mathrm{H}_{9}, \mathrm{C}_{15} \mathrm{H}_{25}, \mathrm{C}_{10} \mathrm{H}_{17}\right)$.

A partir dos modelos representados na Fig. 1, alterase a ramificação lateral, por quatro derivados de óleos essenciais: Prenila, Farnesila, Geranila e seu isômero Nerila.

\section{Resultados e Discução}

Neste trabalho são apresentados os resultados para somente um dos doze complexos, cuja estrutura é a mais simples (MOD1-R=C5H9), os outros complexos seguem o mesmo comportamento. Os complexos estudados apresentam estrutura quadrático plana, os ligantes cloro e amino ligados diretamente ao átomo de platina de forma análoga a cisplatina, conforme ilustra a Fig. 2. 


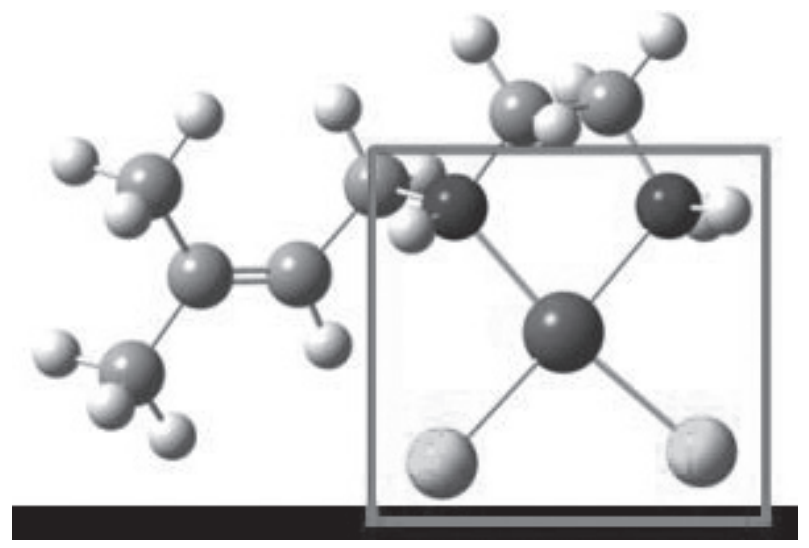

Figura 2 - Representação da estrutura de mínimo local para o complexo $\left(\mathrm{MOD} 1-\mathrm{R}=\mathrm{C}_{5} \mathrm{H}_{9}\right)$.

Os processos reacionais dos complexos apresentam uma etapa de substituição a mais (em relação à cisplatina) devido a não simetria destes complexos. Assim a entrada do ligante aquo fornece propriedades diferentes ao complexo, conforme a posição que este ligante ocupa no lugar do cloro. As reações envolvidas estão descrita pela Fig. 3.

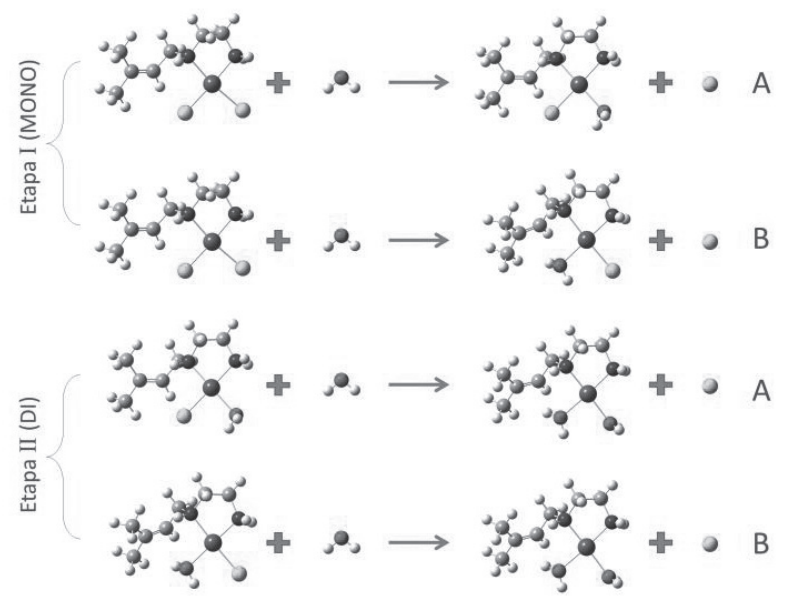

Figura 3 - Equação representante da substituição dos ligantes cloro por ligantes aquo. Observa-se que os complexos não são simétricos como a cisplatina o que realiza a adição da etapa B ao processo, sendo que a cisplatina segue apenas a etapa $\mathrm{A}$.
Após a obtenção dos parâmetros termodinâmicos dos complexos de forma isolada, identificou os estados de transição para cada reação. Os estados de transição obtidos possuem estrutura bipirâmide trigonal, assim como o TS da cisplatina e como esperado para compostos quadráticos planos. Os TS estão ilustrados pela Fig. 4.

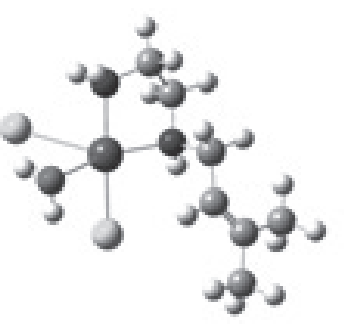

1-A
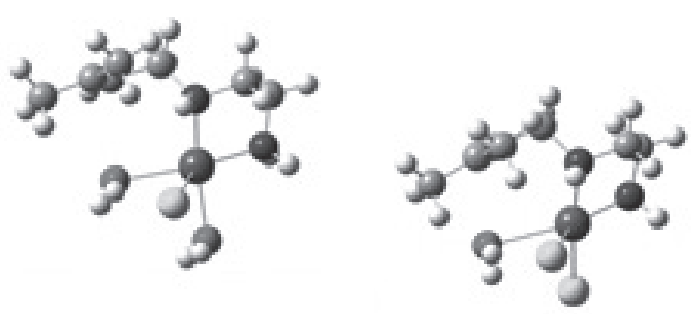

2-A

\section{$1-B$}

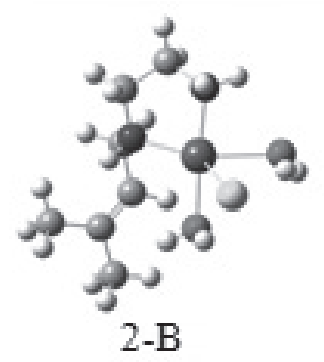

Figura 4 - Estruturas do estados de transição do modelo 1 com ramificação $\mathrm{R}=\mathrm{C}_{5} \mathrm{H}_{9}$, cuja estrutura é bipirâmide trigonal nas quatro etapas reacionais.

Em seguida com os cálculos de IRC, verificouse como ocorreu a substituição dos ligantes, ou seja, o caminho percorrido por cada ligante. Confirmando que os TS pertencem às reações descritas, fornecendo a estrutura dos reagentes e produtos intermediários. Os cálculos de IRC estão demonstrados pela Fig. 5. 
Por fim, otimizando os produtos e reagentes obtidos pelo IRC determinou os parâmetros termodinâmicos e cinéticos envolvidos na reação com reagente e produtos intermediários. A energia de ativação possui um módulo inferior ao da cisplatina, assim como a energia livre de Gibbs. Verifica-se que para o caso dos reagentes e produtos isolados tem-se um absurdo, isto porque os produtos possuem energia superior ao estado de transição, devido ao desbalanceamento de cargas da reação, fato também observado para o processo reacional da cisplatina. Outro fator é não ter adicionado o efeito solvente para descrever melhor o sistema biológico.

As barreiras de energia para as etapas estão ilustradas pela Fig. 6. Para os 12 complexos analisados, tem-se uma energia de ativação em torno de $15 \mathrm{kcal}$. mol $^{-1}$ e cerca de 3 kcal.mol-1 a menos que a cisplatina, sendo um fator significante na velocidade de reação, pois a velocidade de reação é descrita pela equação de Arrhenius ${ }^{5}$, ou seja, esta descrita por uma exponencial, logo torna a velocidade de reação mais sensível a qualquer variação na energia de ativação.

É observado para alguns complexos (etapa 2-A da Fig. 6) uma energia de ativação superior a da cisplatina, com 1,7 kcal.mol ${ }^{-1}$, no entanto em sua maioria os complexos apresentam menor energia de ativação, ou seja, maior velocidade de substituição que a cisplatina.

Um exemplo é o complexo apresentado ao longo do trabalho (MOD1 $\mathrm{R}=\mathrm{C}_{5} \mathrm{H}_{9}$ ) que apresenta a menor energia de ativação para a primeira etapa de substituição (monoaquo) com energia de 11,9 kcal. $\mathrm{mol}^{-1}$.

Para a segunda etapa de substituição o complexo com maior velocidade de substituição é o complexo MOD3 $\mathrm{R}=\mathrm{C}_{15} \mathrm{H}_{25}$.

Em relação a variação da energia livre de Gibbs observa-se que o complexo MOD3 $\mathrm{R}=\mathrm{C}_{15} \mathrm{H}_{25}$ é o mais espontâneo (mais negativo) e para a segunda etapa, tem-se o MOD3 $\mathrm{R}=\mathrm{C}_{10} \mathrm{H}_{17}$, com energia de 88,77 kcal. $\mathrm{mol}^{-1} \mathrm{e} 168,63 \mathrm{kcal}^{\mathrm{mol}} \mathrm{m}^{-1}$ respectivamente.

Reforçando o fato de que a análise com os reagentes e produtos intermediários descrevem melhor a reação, por eliminar o desbalanceamento de cargas, entretanto a otimização e analise vibracional dos reagentes e produtos do IRC ainda estão em andamento.
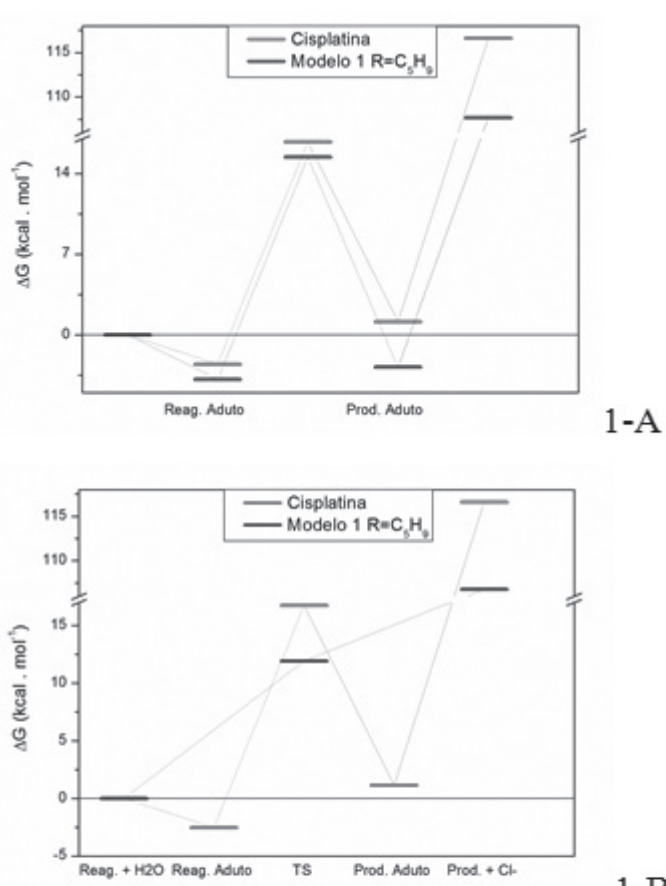

$1-\mathrm{B}$

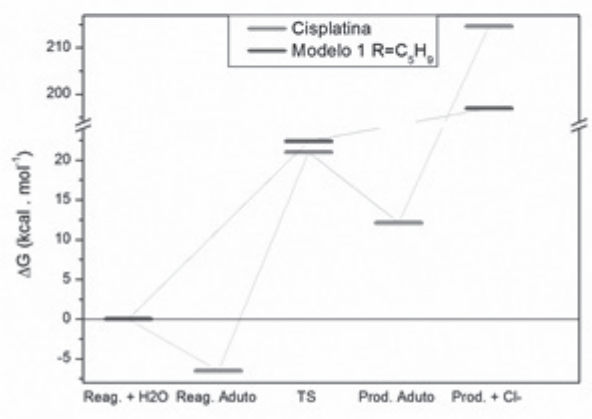

2-A

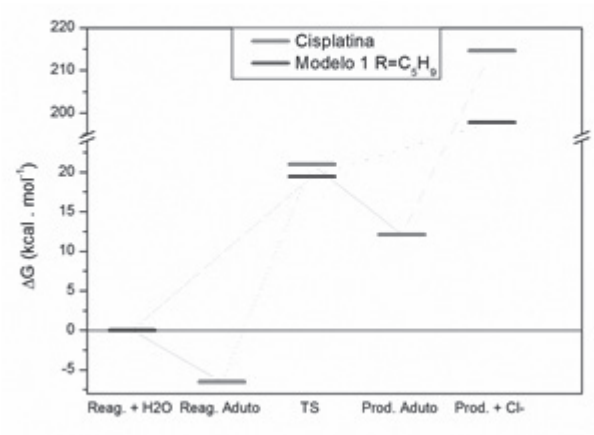

2-B

Figura 6. Comparação dos parâmetros termodinâmicos da cisplatina4 (vermelho) com os complexos (azul), em específico para o modelo 1 $\mathrm{R}=\mathrm{C} 5 \mathrm{H} 9$. 


\section{Conclusão}

Os parâmetros termodinâmicos para os reagentes e produtos intermediários descrevem melhor a reação, por não possuírem o desbalanceamento de carga.

Os parâmetros termodinâmicos dos complexos estão melhorados em relação a cisplatina, pois a energia de ativação é menor e a energia livre de Gibbs mais negativa, o que fornece uma reação mais rápida e mais espontânea.

\section{Agradecimentos}

Os autores são gratos ao suporte dado pelas agencias de fomento FAPEMIG, CNPQ e RQ-MG.

\section{Referências}

1. Lopes, J.F., et al., Theoretical study of the potential energy surface for the interaction of cisplatin and their aquated species with water. Journal of Chemical Physics, 2008. 128(16): p. 14.

2. Lippard, E.R.J.a.S.J., Structure, Recognition, and Processing of Cisplatin-DNA Adducts. Chem. Rev, 1999. 99: p. 2467-2498.

3. Wilson, J.J., J.F. Lopes, and S.J. Lippard, Synthesis, Characterization, and Photophysical Properties of Three Platinum(II) Complexes Bearing Fluorescent Analogues of the Di-2-pyridylmethane Ligand. Inorganic Chemistry, 2010. 49(11): p. 5303-5315.

4. Kai-Chi Lau, J. and Deubel, D. V., Hydrolysis of the Anticancer Drug Cisplatin: Pitfalls in the Interpretation os Quantum Chemical Calculations . Journal of Computational Chemistry. 2005, 2, p. 103-106.

5. Cramer, C., J., Essentials of Computational Chemistry: teories and models. $2^{\mathrm{a}}$ edition. England: John Wiley \& Sons Ltd, 2004.

\section{Lucas Chuman Santan \& Juliana Fedoce Lopes}

LaQC- Laboratório de Química Computacional, Universidade Federal de Itajubá, Av. BPS nº1303, Bairro: Pinheirinho, 37500-903, Itajubá, MG, Brasil 